\title{
Risk Factors of Ischemic Stroke in Children with Head and Neck Tumors: A Clinical Case Description with a Literature Review
}

\author{
Anastasia D. Rodina ${ }^{1}$, Vladimir Sh. Vanesyan ${ }^{1}$, Tatiana V. Gorbunova ${ }^{1,2,3}{ }^{\text {, Natalia V. Ivanova }}{ }^{1}$, \\ Vladimir G. Polyakov $1,2,3$ \\ ${ }^{1}$ Children's Oncological Department of Head and Neck Tumors, N. N. Blokhin National Medical Research Center of Oncology, Moscow, \\ Russian Federation \\ ${ }^{2}$ Department of Pediatric Oncology Named After Academician Lev Abramovich Durnov, Russian Medical Academy of Continuing \\ Professional Education, Moscow, Russian Federation \\ ${ }^{3}$ Department of Otorhinolaryngology, Faculty of Pediatrics, Pirogov Russian National Research Medical University, Moscow, Russian \\ Federation
}

Email address:

dr.rodinaAD@yandex.ru (A. D. Rodina), vanesyan96@mail.ru (V. Sh. Vanesyan), wasicsol@mail.ru (T. V. Gorbunova), nv.ivanova6@gmail.com (N. V. Ivanova),vgp-04@mail.ru (V. G. Polyakov)

\section{To cite this article:}

Anastasia D. Rodina, Vladimir Sh. Vanesyan, Tatiana V. Gorbunova, Natalia V. Ivanova, Vladimir G. Polyakov. Risk Factors of Ischemic Stroke in Children with Head and Neck Tumors: A Clinical Case Description with a Literature Review. American Journal of Pediatrics. Vol. 7, No. 3, 2021, pp. 163-169. doi: 10.11648/j.ajp.20210703.24

Received: August 10, 2021; Accepted: August 24, 2021; Published: August 31, 2021

\begin{abstract}
Ischemic stroke (AI) is becoming an increasingly important and urgent problem in pediatrics due to the occurrence of neurological disorders that affect the quality of life and limit the social adaptation of a child. The clinical picture of ischemic stroke in pediatric practice differs from the manifestation of this pathology in adult patients. Children have special risk factors for the development of IS. In pediatric oncology, there are such factors like invasive tumor growth, the development of tumor embolism, and blood clotting disorders. Side effects of anticancer treatment occupy a special place in the development of IS. The relationship between post-radiation arteriopathies and ischemic stroke has been more studied among cancer patients who underwent neck irradiation. In children, it was found that after radiation therapy to the head and neck region and, thus, the risk of ischemic stroke in childhood increases. This review is devoted to the possible pathophysiological causes and mechanisms of stroke in children with head and neck tumors. In addition, descriptions of clinical cases of patients with ischemic stroke in the course of anticancer treatment are presented.
\end{abstract}

Keywords: Children's Arterial Ischemic Stroke, Chemotherapy, Radiation Therapy, Pediatric Oncology

\section{Introduction}

Ischemic stroke (IS) is an acute violation of cerebral circulation. IS in the pediatric population is rare - 1-6 cases per 100,000 children [1]. Population studies of ischemic stroke in children (aged 29 days to 18 years) show an annual incidence of 2.4 cases per 100,000 people, with a mortality rate of $4 \%$. More than $50 \%$ of the survivors suffer from persistent neurological, cognitive and/or mental disorders. Data on the incidence of ischemic stroke in Russia are extremely scarce. In 2009, V. P. Zykov presented data on the incidence of ischemic stroke in Moscow - 0.79 per 100,000 children per year. According to the published data of $\mathrm{O}$. A. Lvova et al, in 2013, mortality due to IS in children is 7 $28 \%$. Epilepsy occurs in nearly $30 \%$ of stroke survivors within the first decade.

As the survival rates of children with malignant tumors have improved, the long-term effects of cancer therapy are coming to the fore. The relationship between ischemic stroke and cancer has attracted the attention of the medical community since a long time. Researchers from The Netherlands Cancer Institute, (Amsterdam, Netherlands) published data on the overall five-year survival of patients with Hodgkin's lymphoma - 7\% of patients developed 
ischemic stroke during a 17.5-year follow-up period [2]. The relationship between arteriopathies and ischemic stroke has been more studied among cancer patients who underwent neck irradiation. In children, it was found that after radiation therapy to the head and neck region and, thus, the risk of ischemic stroke in childhood increases. A few studies of this pathology in children have shown that patients with the tumor localized in the region of the base of the skull, the brain, who underwent chemoradiation therapy, are at risk of developing ischemic stroke [3]. In this case, the main reason is associated with arteriopathies developing in the early postradiation period [4]. Atherosclerosis is the most common cause of ischemic stroke in adult patients who have received head and neck radiation therapy [5].

At the International Conference on Stroke and 54 annual Meeting (2012) of the American Society of Radiation Oncology (ASTRO) in Boston, the data from a retrospective cohort study of 14,358 children with cancer survivors was presented. The results prove that children with tumors of the central nervous system (CNS), head and neck, acute lymphoblastic leukemia are at risk of developing a stroke [6, 7]. The main pathophysiological mechanisms of the development of stroke in cancer patients are associated both directly with the oncological disease itself and with complications of anticancer treatment - blood clotting disorders, the development of infection, and/or therapeutic and diagnostic procedures [8]. Cases of ischemic stroke caused by coagulopathy have been described. Blood clotting disorders are the most common cause of cardiovascular disease in cancer patients and include disseminated intravascular coagulation (DIC), non-bacterial thrombotic endocarditis (NBTE) and thrombocytopenia [9].

Infectious processes are one of the main causes of stroke. The relationship between the development of ischemic stroke and systemic infection take place due to the development of a systemic inflammatory response, due to damage to the vascular endothelium and abnormal dilatation. The medical literature describes infections caused by bacteria (Helicobacter pylori, Chlamydia pneumoniae, Mycoplasma pneumoniae, Haemophilus influenzae, Staphylococcus aureus and viridans), fungi (Cryptococcus spp., Candida spp., Aspergillus spp., Epstein virus herpes simplex -1, -2, as well as cytomegalovirus), which led to the development of IS [10].

In clinical practice, ischemic stroke associated directly with a tumor process, which is caused by invasion of arterial and/or venous sinuses, compression of blood vessels by a tumor component, leptomeningeal metastasis, and tumor embolism [11]. One of the causes of ischemic stroke is a tumor embolism of the cerebral vessels. The source of tumor emboli brought by the blood flow is most often the heart (myxomas or other tumors of the heart and lungs). Large emboli can occlude the middle cerebral artery trunk and lead to massive brain damage involving the cortex, gray and white matter. The focus of ischemic stroke is formed when an embolus occludes a small feeding branch of the middle cerebral artery or basilar artery [12]. Cavernous sinus thrombosis interferes with venous outflow, which leads to the development of intracranial hypertension, which results in cerebral ischemia [13]. Risk factors for cavernous sinus thrombosis are the development of post-radiation otitis and/or rhinosinusitis, trauma, and concomitant hereditary thrombophilia [14]. Cases of IS associated with thrombosis of the cavernous sinus (lesions of the superior sagittal sinus were more often observed) were identified in a retrospective study by Santoro N. et al., 2005, which included 2318 children with acute lymphoblastic leukemia (ALL). The incidence of ischemic stroke in this cohort of patients was $0.47 \%$ [15].

Leptomeningeal metastasis and / or damage to the brain substance develops in 5\% of cases in children with sarcomas of paramenengial localization, nasopharyngeal cancer, neuroblastoma, lymphoma and malignant melanoma. Strokes in this cohort of patients result from infiltration of the vessel walls of the brain. Multiple metastatic brain damage leads to occlusion of the venous sinus, which provokes the development of ischemic stroke [16]. Tumor growth in the Virchow-Robin perivascular spaces promotes the development of thrombosis, wall infiltration and vascular spasm, which further leads to the development of ischemic brain damage [17].

The occurrence of ischemic stroke is closely related to anticancer therapy - systemic chemotherapy and/or radiation therapy to the head and neck area. The mechanisms by which anticancer agents lead to stroke include endothelial abnormalities and clotting factor abnormalities, as well as the development of immunosuppression and opportunistic infections. In addition, it has been reported about the development of ischemic stroke as a result of the effect of chemotherapy on the hemostatic system [18].

Systemic chemotherapy with 5-fluorouracil, cisplatin, carboplatin, cyclophosphamide, paclitaxel, L-asparaginase can cause cerebrovascular disorders that lead to the development of ischemic stroke regardless of the morphological variant of cancer [19]. A retrospective study conducted at Chang Gung Memorial Hospital (Taiwan, 2006) showed that a potential risk of stroke occurs in patients who received cisplatin. In this case, there is a decrease in the levels of circulating endothelial and platelet microparticles [20]. Microparticles of endothelial origin are biological markers of endothelial damage, they are involved in the pathogenesis of cardiovascular and metabolic diseases, and reflect the balance between proliferation and apoptosis. Rollins N, et al. reports isolated cases of diffuse reversible arterial disorders, corresponding to vasospasm, with acute neurotoxicity after intrathecal injection of methotrexate [21].

Complications of chemotherapy, radiation therapy and hematopoietic stem cell transplantation can occur both during treatment and after treatment [22]. They are manifested by damage to the endothelium and impaired blood coagulation, the addition of opportunistic infections as a result of immunosuppression. In addition, it has been reported about the development of ischemic stroke as a result of the direct effect of chemotherapy on the hemostatic system [18].

Radiation reactions have natural and inevitable 
consequences of irradiation of healthy organs and tissues during radiation therapy on the head and neck region. The general radiation response of the body is associated not only with the direct effect of radiation, but also with intoxication with tumor decay products and direct damage to the cellular elements of vital organs. In the presented cohort studies of patients with various solid and hematological tumors who received radiation therapy to the head region, an increased risk of developing non-atherosclerotic radiation-induced cerebral vasculopathy due to stenosis of the internal and/or common carotid artery was described. The incidence of radiation-induced secondary stenosis of the internal carotid artery ranges from $12 \%$ to $60 \%[5,11,22]$. According to Scott et al., 2009, the risk of stroke increases by $2.6 \%$ after radiation therapy to the neck area [23]. Fouladi et al. reports that patients of early childhood (under five years old) at the time of radiation therapy are more susceptible to the development of strokes, due to vasculopathy of small vessels [24].

The main pathophysiological mechanism of the damaging effect of radiation therapy, which increases the risk of stroke, is not fully understood. The occurrence and degree of these changes depend on the phase of the radiation exposure. The reaction of normal tissues, induced by radiation, depends on the size of the fraction (the value of a single dose), the magnitude of the absorbed dose, its power, type of radiation, and the volume of radiation [25].

The mechanism of radiation-induced cell damage - chronic oxidative stress has a sharp increase in oxidative processes in the body with insufficient functioning of the antioxidant system. The signal for starting this reaction is a change in the intracellular environment, leading to a shift in the equilibrium of the concentrations of prooxidant and antioxidant components (an increase in the production of reactive oxygen species, causing damage to proteins and lipids) with the subsequent activation of oxidation processes. Activation of this process leads to chronic inflammation, increasing the risk of development and rupture of atherosclerotic plaques - accelerated early atherosclerosis through chronic inflammation within a year after treatment [26].

A recent study by Lee CW et al. (2013), based on a large cohort of cancer patients with malignant neoplasms, demonstrated that symptomatic morphine therapy of cancer patients can also potentiate stroke [27].

\section{Clinical Case \# 1}

Under our supervision was a boy A, 14 years old, diagnosed with "Undifferentiated nasopharyngeal cancer, metastatic lesion of the lymph nodes of the neck on both sides. T4N2M0. Stage IV." N. Blokhin, Moscow (hereinafter-Scientific Research Institute).

Anamnesis of life. A child from a second physiological pregnancy, second independent childbirth at a period of 40 weeks. Birth weight $-3000 \mathrm{~g}$, height $-52 \mathrm{~cm}$. According to the Apgar scale - $8 / 9$ points. The neonatal period was uneventful. Past diseases: acute respiratory infections. Vaccination according to the National Vaccination Schedule. Cancer history is not burdened. There were no malignant neoplasms in the family or in the immediate family of the child.

Medical history. From the history of the disease, it is known that the first manifestation of the disease was in May 2020 in the form of a seal in the left behind the ear region. There was a gradual increase in pain in the left ear, in connection with which they turned to a pediatrician and an otorhinolaryngologist at the place of residence. During the observation, an increase in the titer of antibodies to the early antigen of the Epstein-Barr virus (EBV EA-IgG Ab) up to 600 units was noted. On the basis of a comprehensive examination, the diagnosis was made: "Infectious mononucleosis of moderate severity, typical form", symptomatic therapy was recommended. In July 2020, appeared a sharp violation of nasal breathing, trismus of the masticatory muscles. According to the MRI of the skull and the brain was revealed a volumetric neoplasm of the nasopharynx with damage to the lymph nodes of the neck on both sides. For the purpose of morphological verification, was performed a biopsy of the nasopharyngeal neoplasm. According to the results of the histological conclusion undifferentiated cancer of the nasopharynx. The patient is referred for consultation and determination of treatment tactics at the Research Institute. In the conditions of the Research Institute of N. Blokhin, on the basis of a comprehensive examination was made a clinical diagnosis: "Undifferentiated nasopharyngeal cancer, metastatic lesion of the lymph nodes of the neck on both sides. T4N2M0. Stage IV". The child received four courses of chemotherapy with vinblastine at a dose of $5 \mathrm{mg} / \mathrm{m}^{2}$, cyclophosphamide at a dose of $600 \mathrm{mg} / \mathrm{m}^{2}$, doxorubicin at a dose of $20 \mathrm{mg} / \mathrm{m}^{2}$, bleomycin at a dose of $10 \mathrm{mg} / \mathrm{m}^{2}$ according to the clinical guidelines for the treatment of nasopharyngeal cancer (2017). In connection with the stabilization of the tumor process (reduction of the main tumor focus by $25 \%$ ) after induction chemotherapy, it was decided to conduct 5 courses of second-line chemotherapy with cisplatin at a dose of $100 \mathrm{mg} / \mathrm{m}^{2}$, paclitaxel at a dose of $150 \mathrm{mg} / \mathrm{m}^{2}, 5$-fluorouracil at a dose of $1000 \mathrm{mg} / \mathrm{m}^{2}$. After the end of the 3 rd course of chemotherapy of the 2 nd line, the child had tonic-clonic seizures. In the first 24 hours was performed MRI of the brain with contrast and there was revealed MRI pattern of ischemic stroke in the cortex of both hemispheres of the brain was revealed as a multifocal lacunar lesion (Figure 1).

Anticonvulsant (convulex), anticoagulant (low molecular weight heparin), decongestant (dexamethasone) and symptomatic therapy were performed. The child's condition after 72 hours with positive dynamics in the form of normalization of the general condition of the child and indicators of the coagulogram.

At moment of writing this article the patient is clinically stable and without neurological deficits. Antitumor therapy was continued according to the plan - two courses of chemotherapy with second-line drugs were carried out and 
radiation therapy (total focal dose $=55.4 \mathrm{~g}$ ) was carried out against the background of the use of oral anticoagulants under the control of coagulogram, anticonvulsant therapy with valproic acid.

According to the results of genetic analysis, polymorphism of the genes of the factors of the hemostasis system was revealed, which caused their abnormal synthesis or impaired functional activity. The following mutations were found in the F5 gene: Factor V Leiden (genetic markers G1691A; Arg506Gln); polymorphism in the ITGA2 gene (genetic marker C807T), also revealed a polymorphism in the SERPINE1 gene in $4 \mathrm{G} / 5 \mathrm{G}$ alleles, a homozygous mutation in the MTR gene (genetic marker A2756G), a homozygous mutation in the MTRR gene (genetic markers Ile22Met and A66G). Therefore, the revealed structural changes in the genes of the hemostasis system led to an imbalance between prothrombotic and anti-thrombotic factors of blood coagulation - congenital thrombophilia. The presence, in this case, of a rare allelic variant of coagulation factor $\mathrm{V}$ (Leiden mutation) suggests a high risk of thrombus formation, even without the involvement of external provoking factors.

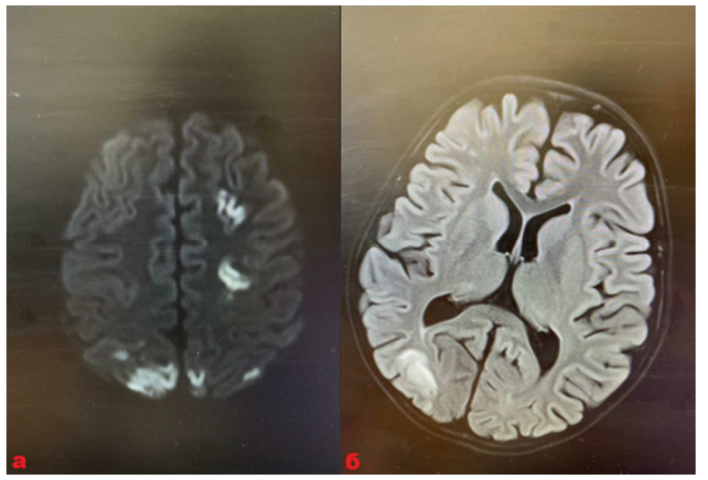

Figure 1. MRI picture of ischemic stroke in the cortex of both hemispheres of the brain (frontal, occipital, parietal lobes) by the type of multifocal lacunar lesion in DWI mode (a), in T2Flair mode (b).

\section{Clinical Case \# 2}

We observed a 3 years old girl A, with a diagnosis of "Alveolar rhabdomyosarcoma of parameningeal localization with intracranial spread, metastatic lesions of the lymph nodes of the neck on both sides. Stage T2N1M0. Art. III".

Anamnesis of life. A girl from a third physiological pregnancy, a third operative delivery at 38 weeks. Birth weight - $3890 \mathrm{~g}$, height $-51 \mathrm{~cm}$. According to the Apgar scale $-8 / 9$ points. The neonatal period was uneventful. She grew and developed with age. Past diseases: acute respiratory infections. Vaccination according to the National Vaccination Schedule. Cancer history is not burdened. Malignant neoplasms in the family and in the closest relatives of the child were not identified

Medical history. From the anamnesis of the disease it is known that the mother considers the child sick since August 2017, when appeared discharge with an admixture of blood from the nasal passages. A pediatrician and otolaryngologist at the place of residence observed the girl. She got symptomatic and antibiotic therapy with a positive effect. In November 2017, a sharp negative trend was noted in the form of a complete absence of nasal breathing, the appearance of exophthalmos, and an increase in weakness. The child was urgently hospitalized in the children's surgical department of the regional hospital. According to the CT scan of the skull was revealed a volumetric neoplasm of the nasal cavity. Tumor biopsy was performed. According to the results of histological examination rhabdomyosarcoma was diagnosed. The patient was referred for consultation and determination of treatment tactics at the Research Institute of N. Blokhin. Under the conditions of the Research Institute, on the basis of a comprehensive examination, a clinical diagnosis was made: "Alveolar rhabdomyosarcoma of parameningeal localization with intracranial spread, metastatic lesion of the lymph nodes of the neck on both sides. Stage T2N1M0. St. III." $1.5 \mathrm{mg} / \mathrm{m}^{2}$, cyclophosphamide $1200 \mathrm{~m} / \mathrm{m}^{2}$, doxorubicin $37.5 \mathrm{mg} / \mathrm{m}^{2}$, ifosfamide 1800 $\mathrm{mg} / \mathrm{m}^{2}$, etoposide $100 \mathrm{mg} / \mathrm{m}^{2}$, irinotecan $50 \mathrm{mg} / \mathrm{m}^{2}$, dactinomycin $0.045 \mathrm{mg} / \mathrm{kg}$ and radiation therapy to the area of primary tumor location and lymph nodes of the neck on both sides (FD - 45.0g). After 14 courses of chemotherapy, 3 months after radiation therapy the child developed an episode of generalized clonic seizures, left-sided hemiplegia. The development of convulsive syndrome revealed MR picture of impaired cerebral circulation in the basin of the right middlecerebral artery. (Figure 2).

For a month the child underwent anticonvulsant (convulex), anti-edematous (dexamethasone), anticoagulant (low molecular weight heparin), nootropic and symptomatic therapy on the basis of a specialized neurological hospital. During treatment, the child had a persistent neurological deficit. Due to timing violation and contraindication to chemotherapy, the treatment was completed.

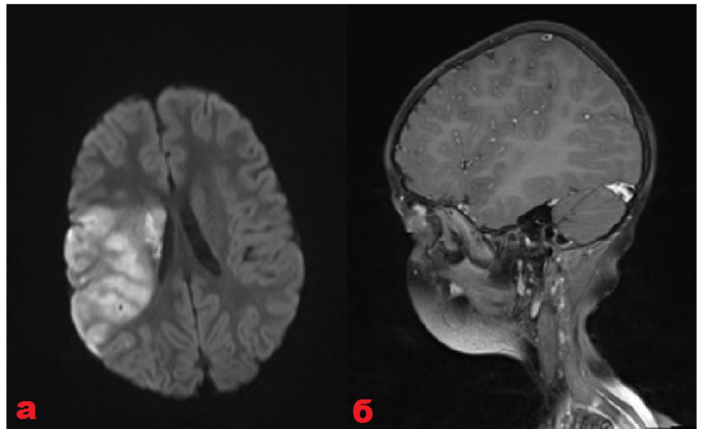

Figure 2. MR - signs of ischemic damage to the right temporal, parietal lobes and shell, corpus callosum in the form of an area of limited diffusion on DWI in axial projection (a), in sagittal projection in T1 mode contrast (b).

\section{Discussion}

This article summarizes the pathophysiological and clinical characteristics of ischemic stroke in children. Tumors localized in the head and neck region can cause ischemic stroke by direct external occlusion of the cerebral vessels. In the presented clinical cases, the tumors were localized parameningeally. In the first case, the tumor was located in 
the nasopharyngeal cavity, completely overlapping the choanal gap and spreading to the left nasal passage, left pterygoid fossa; intracranial spread was not observed. In the second case, the tumor occupies a median position, destroyed the middle and posterior cells of the ethmoid labyrinth, spread into the orbits, maxillary sinuses, into the pterygopalatine and pterygoid fossa from 2 sides, the intracranial component of the tumor was determined in the region of the anterior and middle cranial fossa.

Studies [18-20] have shown that chemotherapy is a powerful risk factor for the development of cerebrovascular disease. Currently, however, the pathophysiology of chemotherapy-induced stroke is not entirely clear. Violations in the hemostasis system during chemotherapy promotes thrombus formation (hypercoagulation is the cause of stroke in children in $2-7 \%$ of cases). It leads to thrombosis of the subclavian, jugular veins, deep veins of the leg, migratory thrombophlebitis (Trusso's syndrome), pulmonary embolism, cerebral vein thrombosis. The cause of hypercoagulation is often multifactorial: increased coagulability, damage to the walls of blood vessels, emboli, damage to the endothelium due to chemotherapy, vegetation with non-bacterial endocarditis, the effect of the tumor on adjacent tissues. Arterial thrombosis is associated with damage to the endothelium, increased platelet activity. In clinical practice there are intracranial tumor spread, leukostasis, increased platelet aggregation lead to thrombosis of cerebral vessels; cases of venous thrombosis due to neuroblastoma metastases have been described.

In both cases, the patients underwent polychemotherapy, which included vinakaloids, cyclophosphamide, doxorubicin, and platinum preparations. The combination of the localization of the tumor process with the undesirable effects of chemotherapy can be considered as a co-factor synergistic effect on the vascular wall.

In the second case, the child underwent irradiation of the skull base tumor, FD of 55.2g and lymph nodes of the neck on both sides, FD of $45.0 \mathrm{~g}$. Studies [23] have shown that radiation therapy significantly increases the risk of transient ischemic attack and ischemic stroke. To date, the scientific community has limited information about the timing and frequency of radiation-induced ischemic stroke, which is based on endothelial damage with the accumulation of foam cells, perivascular lymphocytic infiltration and interstitial fibrosis. In large vessels (carotid arteries and branches of the circle of Willis) develops fibrosis of the vascular wall. The interval from the start of radiation therapy to the onset of symptoms depends on the diameter of the vessel - the larger the diameter, the later the symptoms appear. Radiation vasculopathy of the intracranial vessels is often seen in young patients. Symptoms may manifest for several months after treatment. There is no evidence-based recommendation for the treatment of asymptomatic and symptomatic radiation-induced vasculopathy.
Predisposing factors such as thrombophilia, congenital or acquired heart and central nervous system defects have been noted to increase the risk of ischemic stroke. The International Pediatric Stroke Study (IPSS) reports on the main systemic factors underlying childhood stroke, in particular: sickle cell disease (SCD), congenital heart defects, trauma and infections such as meningitis, sepsis and encephalitis. In the first case, the results of genetic analysis revealed mutations in the F5 gene: Factor V Leiden, polymorphism in the ITGA2 gene - congenital thrombophilia The presence of a rare allelic variant of the Leiden mutation suggests a high risk of ischemic stroke. In the second case, no mutations were found in the genes of the hemostasis system. Probably a predisposing factor in the development of ischemic stroke was radiation therapy to the area of the primary location of the tumor and lymph nodes of the neck on both sides.

Based on the analysis of foreign and domestic medical literature, as well as on the basis of own experience, Figure 3 presents the causes of ischemic stroke in the form of a flowchart.

The clinical picture and risk factors for ischemic stroke in children with cancer are different from adults, and depend on the presence of genetic changes in the coagulation system, tumor localization, its intracranial spread, drug antitumor therapy and radiation therapy, leading to disorders in the coagulation system [18]. In addition, specific anticancer therapy is accompanied by various complications, such as the development of hypercoagulability and infection.

One of the main problems faced by pediatricians and pediatric oncologists is the differential diagnosis of acute neurological disorders. Symptoms such as headache, seizures, impaired level of consciousness are found in a wide range of diseases. Therefore, it is necessary to collect the anamnesis, clinical and instrumental - laboratory examinations carefully, taking into account the predisposing factors. Because the prognosis and mortality in ischemic stroke depend on timely and adequate diagnosis, this problem becomes more urgent. In children, are often recorded focal neurological disorders, such as movement disorders, convulsive syndrome, speech impairment. The middle cerebral artery is most often affected [12]. According to Fullerton et al. (2016) the development of convulsive syndrome on the first day after a stroke increases the risk of developing epilepsy in the future [28].

High compensatory abilities of the brain of children make it possible to achieve complete restoration of impaired functions. To reduce mortality and disability, it is important to use an interdisciplinary approach to treat this cohort of patients with the involvement of neurologists, pediatricians, and hematologists. Long-term prospective studies in this cohort of patients remain a priority, as the incidence of this problem will probably help to increase with improved survival. $[7,10,27]$ 


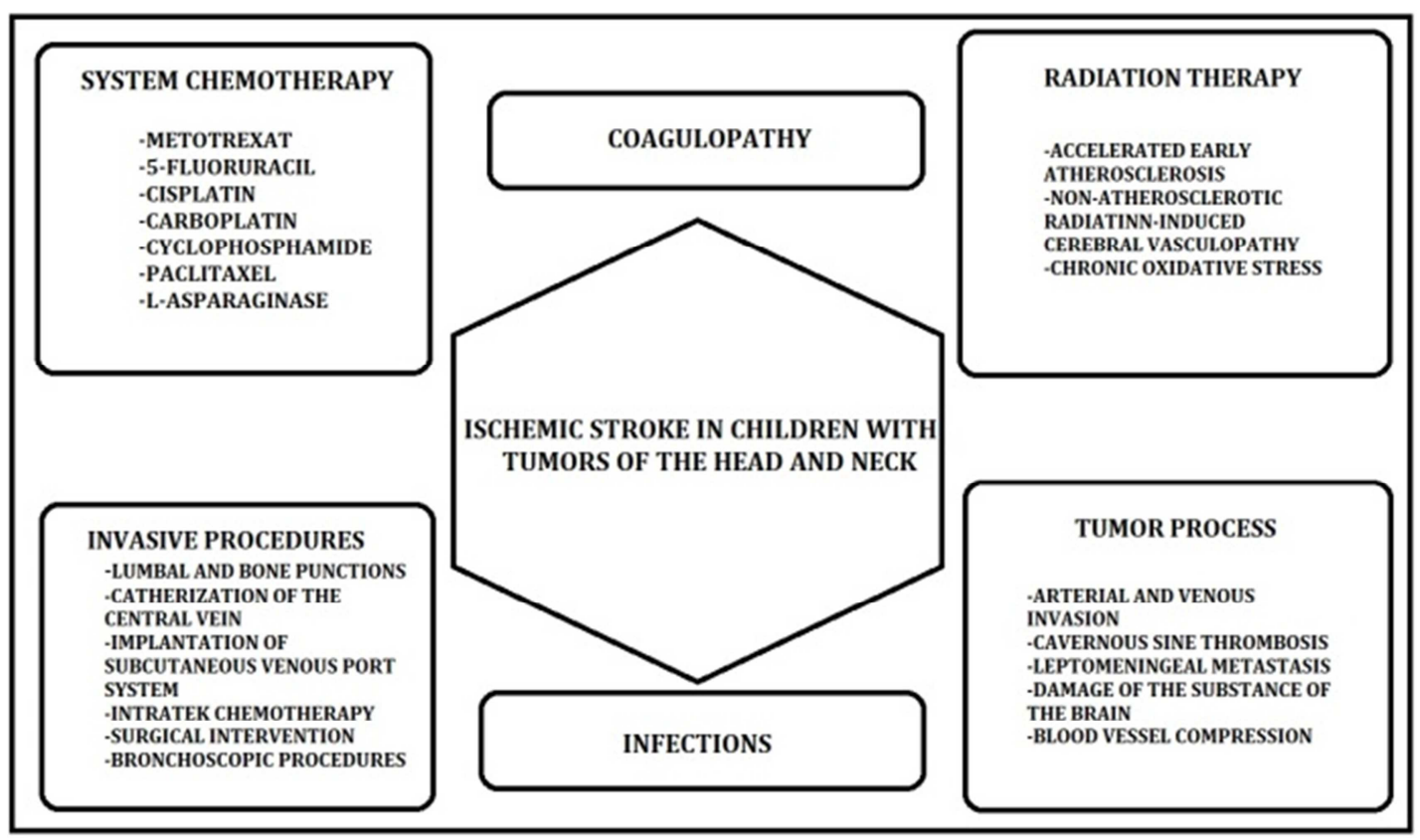

Figure 3. Main pathophysiological mechanisms of development ischemic stroke in children with head and neck tumors.

\section{Conclusion}

In conclusion, it should be noted that pediatric patients receiving treatment for cancer have not only an increased risk of stroke, but also a high risk of recurrent stroke. The Children's Oncology Group (COG) has developed guidelines for screening survivors at risk of persistent or late effects of cancer therapy. The COG currently recommends an annual neurologic examination for all patients who have received more than 18 Grays of cranial radiation therapy. During dispensary observation should be taken preventive measures to reduce the influence of risk factors for the development of cerebrovascular diseases (stroke and / or transient ischemic attack) in these patients. Duplex ultrasound examination is recommended for patients with head and neck tumors who have received specific therapy.

\section{References}

[1] Margherita Rosa, Silvana De Lucia. "Paediatric arterial ischemic stroke: acute management, recent advances and remaining issues" Ital J Pediatr. 2015; 41: 95. Published online 2015 Dec 2. doi: 10.1186/s13052-015-0174-y.

[2] De Bruin ML, Dorresteijn LD, van't Veer MB, Krol AD, van der Pal HJ, Kappelle AC, Boogerd W, Aleman BM, van Leeuwen FE. Increased risk of stroke and transient ischemic attack in 5-year survivors of Hodgkin lymphoma. $J$ Natl Cancer Inst. 2009; 101: 928-937. doi: 10.1093/jnci/djp147.

[3] Omura M, Aida N, Sekido K, et al. Large intracranial vessel occlusive vasculopathy after radiation therapy in children: clinical features and usefulness of magnetic resonance imaging. Int J Radiat Oncol Biol Phys. 1997; 38: 241-249.

[4] Ullrich NJ, Robertson R, Kinnamon DD, et al. Moyamoya following cranial irradiation for primary brain tumors in children. Neurology. 2007; 68: 932-938.
[5] Dorresteijn LD, Kappelle AC, Scholz NM, et al. Increased carotid wall thickening after radiotherapy on the neck. Eur $J$ Cancer. 2005; 41: 1026-1030.

[6] Khachaturov Yu. A., Shchederkina I. O., Plavunov N. F. and others. STROKE IN CHILDREN AND ADOLESCENTS [STKOV: ACTUAL PROBLEMS OF PREHOSPITAL DIAGNOSTICS. Archive of Internal Medicine. 2020; 10 (1): 21-30. DOI: 10.20514/2226-6704-2020-10-1-21-30.

[7] Sabine Mueller, Heather J. Fullerton, Kayla Stratton, Wendy Leisenring, Rita E. Weathers, Marilyn Stovall, Gregory T. Armstrong, Robert E. Goldsby, Roger J. Packer, Charles A. Sklar, Daniel C. Bowers, Leslie L. Robison, Kevin R. Krull/ Radiation, Atherosclerotic Risk Factors, and Stroke Risk in Survivors of Pediatric Cancer: A Report From the Childhood Cancer Survivor Study/International Journal of Radiation Oncology*Biology*Physics, Volume 86, Issue 4, 15 July 2013, Pages 649-655.

[8] Aarnio K, Joensuu H, Haapaniemi E, Melkas S, Kaste M, Tatlisumak T, Putaala J. Cancer in young adults with ischemic stroke. Stroke. 2015; 46: 1601-1606. doi: 10.1161/STROKEAHA.115.008694.

[9] Katz JM, Segal AZ. Incidence and etiology of cerebrovascular disease in patients with malignancy. Curr Atheroscler Rep. 2005; 7: 280-288. Doi: 10.1007/s11883005-0020-6.

[10] Fugate JE, Lyons JL, Thakur KT, Smith BR, Hedley-Whyte ET, Mateen FJ. Infectious causes of stroke. Lancet Infect Dis. 2014; 14: 869-880. Doi: 10.1016/S1473-3099(14)70755-8.

[11] Grisold W, Oberndorfer S, Struhal W. Stroke and cancer: A review. Acta Neurol Scand. 2009; 119: 1-16. doi: 10.1111/j.1600-0404.2008.01059.x.

[12] Zheng Z, Guo G, Xu L, Lei L, Wei X, Pan Y. Left atrial myxoma with versus without cerebral embolism: Length of symptoms, morphologic characteristics, and outcomes. Tex Heart Inst J. 2014; 41: 592-595. Doi: 10.14503/THIJ-133862 . 
[13] Ichord R. Cerebral sinovenous thrombosis. Front Pediatr. 2017; 5: 163. doi: 10.3389/fped.2017.00163].

[14] Ranta S, Tuckuviene R, Mäkipernaa A, Albertsen BK, Frisk T, Tedgård U, Jónsson ÓG, Pruunsild K, Gretenkort Andersson $\mathrm{N}$, Winther Gunnes $\mathrm{M}$, et al. Cerebral sinus venous thromboses in children with acute lymphoblastic leukaemia - a multicentre study from the Nordic Society of Paediatric Haematology and Oncology. Br J Haematol. 2015; 168: 547552. doi: $10.1111 / \mathrm{bjh} .13162$.

[15] Santoro N, Giordano P, Del Vecchio GC, Guido G, Rizzari C, Varotto S, Masera G, De Mattia D. Ischemic stroke in children treated for acute lymphoblastic leukemia: A retrospective study. J Pediatr Hematol Oncol. 2005; 27: 153-157. doi: 10.1097/01.mph.0000157379.44167.b5.

[16] Herman C, Kupsky WJ, Rogers L, Duman R, Moore P. Leptomeningeal dissemination of malignant glioma simulating cerebral vasculitis. Case report with angiographic and pathological studies. Stroke. 1995; 26: 2366-2370. doi: 10.1161/01.STR.26.12.2366] [Raizer JJ, DeAngelis LM. Cerebral sinus thrombosis diagnosed by MRI and MR venography in cancer patients. Neurology. 2000; 54: 12221226. doi: 10.1212/WNL.54.6.1222.

[17] Klein P, Haley EC, Wooten GF, VandenBerg SR. Focal cerebral infarctions associated with perivascular tumor infiltrates in carcinomatous leptomeningeal metastases. Arch Neurol. 1989; 46: 1149-1152. Doi: 10.1001/archneur.

[18] Saynak M, Cosar-Alas R, Yurut-Caloglu V, Caloglu M, Kocak Z, Uzal C. Chemotherapy and cerebrovascular disease. J BUON. 2008; 13: 31-36.

[19] Li SH, Chen WH, Tang Y et al. Incidence of ischemic stroke post-chemotherapy: a retrospective review of 10,963 patients. Clin Neurol Neurosurg 2006; 108: 150-6.

[20] Periard D, Boulanger CM, Eyer S et al. Are circulating endothelial-derived and platelet-derived microparticles a pathogenic factor in the cisplatin-induced stroke? Stroke 2007; 38: $1636-8$.
[21] Rollins N, Winick N, Bash R, Booth T. Acute methotrexate neurotoxicity: Findings on diffusion-weighted imaging and correlation with clinical outcome. AJNR Am J Neuroradiol. 2004; 25: 1688-1695.

[22] Efthimios Dardiotis, Athina-Maria Aloizou, Sofia Markoula, Vasileios Siokas, Konstantinos Tsarouhas, Georgios Tzanakakis, Massimo Libra, Athanassios P. Kyritsis, Alexandros G. Brotis, Michael Aschner, Illana Gozes, Dimitrios P. Bogdanos, Demetrios A. Spandidos, Panayiotis D. Mitsias, Aristidis Tsatsakis Cancer-associated stroke: Pathophysiology, detection and management (Review) Int $\mathrm{J}$ Oncol. 2019 Mar; 54 (3).

[23] Scott AS, Parr LA, Johnstone PA. Risk of cerebrovascular events after neck and supraclavicular radiotherapy: A systematic review. Radiother Oncol. 2009; 90: 163-165. doi: 10.1016/j.radonc.2008.12.019.

[24] Fouladi M, Langston J, Mulhern R, et al. Silent lacunar lesions detected by magnetic resonance imaging of children with brain tumors: a late sequela of therapy. J Clin Oncol. 2000; 18: 824-831.

[25] J. C. Haynes, M. Machtay, R. S. Weber, et al. Relative risk of stroke in head and neck carcinoma patients treated with external cervical irradiation Laryngoscope, 112 (2002), pp. 1883-1887.

[26] H. J. Fullerton, Y. W. Wu, S. Sidney, et al. Risk of recurrent childhood arterial ischemic stroke in a population-based cohort: The importance of cerebrovascular imaging Pediatrics, 119 (2007), pp. 495-501.

[27] Lee CW, Muo CH, Liang JA, Sung FC, Kao CH. Association of intensive morphine treatment and increased stroke incidence in prostate cancer patients: a population-based nested case-control study Jpn J Clin Oncol 2013 Aug; 43 (8): 776-81. doi: 10.1093/jjco/hyt080.

[28] Fullerton H. J., Wintermark M., Hills N. K. et al. Risk of recurrent arterial ischemic stroke in childhood: a prospective international study. Stroke. 2016; 47 (1): 53-9. DOI: 10.1161/STROKEAHA.115.011173. 